\title{
Liver imaging reporting and data system and CT/MRI diagnosis of hepatocellular carcinoma
}

\author{
Devaraju Kanmaniraja, Victoria Chernyak \\ Radiology, Montefiore Medical Center, The Bronx, New York, NY 10467, USA. \\ Correspondence to: Prof. Victoria Chernyak, Radiology, Montefiore Medical Center, The Bronx, 111 East 210th St Bronx, New \\ York, NY 10467, USA. E-mail: vichka17@hotmail.com
}

How to cite this article: Kanmaniraja D, Chernyak V. Liver imaging reporting and data system and CT/MRI diagnosis of
hepatocellular carcinoma. Hepatoma Res2020:6:51. http://dx.doi.org/10.20517/2394-5079.2020.46

Received: 29 Apr 2020 First Decision: 11 Jun 2020 Revised: 21 Jun 2020 Accepted: 24 Jun 2020 Published: 15 Aug 2020

Academic Editor: Yuko Kono Copy Editor: Cai-Hong Wang Production Editor: Jing Yu

\begin{abstract}
The Liver Imaging Reporting and Data System (LI-RADS) is a comprehensive and robust system which provides an algorithmic approach to stratify the probability of hepatocellular carcinoma (HCC) for each observation found in patients at risk for HCC. LI-RADS uses a standardized terminology and approach to improve communication between the radiologist and clinicians. LI-RADS version 2018 is noteworthy for its adoption by the American Association for the Study of Liver Disease into its HCC practice guidance. This manuscript provides an overview of the history of LI-RADS, reviews the Computed tomography/magnetic resonance imaging diagnostic algorithm, highlights the key diagnostic criteria for each category, and discusses the advantage of incorporating LI-RADS in clinical practice.
\end{abstract}

Keywords: Liver Imaging Reporting and Data System, hepatocellular carcinoma, cirrhosis, hepatocellular carcinoma diagnosis

\section{INTRODUCTION}

Hepatocellular cancer (HCC) is the most common primary malignancy of the liver ${ }^{[1]}$. It is the fifth most common cancer in the world and fourth leading cause of cancer mortality ${ }^{[1,2]}$. The incidence of HCC in the United States has been rapidly rising over the last 20 years and predicted to increase until $2030^{[3,4]}$. Imaging plays a critical role in the management of HCC, as the diagnosis of HCC is usually made non-invasively in the appropriate at-risk patient population, based on imaging features alone without the need of pathologic

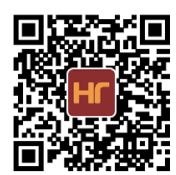


confirmation $^{[s]}$. When biopsy is required, it is often done with image guidance; furthermore, imaging plays an integral role in patients undergoing surveillance or following loco-regional therapy ${ }^{[6]}$. The Liver Imaging Reporting and Data System(LI-RADS) is a comprehensive and dynamic system which provides a standardized means of assessing and communicating the entire spectrum of lesions and pseudolesions in studies performed in patients who are at risk for developing $\mathrm{HCC}^{[7,8]}$. LI-RADS is developed by an international multidisciplinary consortium of radiologists, hepatologists, hepatobiliary surgeons, and hepatopathologists ${ }^{[7,8]}$.

\section{OVERVIEW}

Multiple societies and organizations have proposed image-based systems for the diagnosis of HCC. In 2008, the American College of Radiology proposed the LI-RADS with the primary goal of standardizing the lexicon, interpretation, and reporting of imaging studies specifically in patients who are at risk for developing HCC. The aim was to establish a comprehensive algorithm that would accurately stratify the probability of HCC and malignancy in each observation, while still maintaining a high specificity for $\mathrm{HCC}^{[7]}$. The first version was released in 2011, followed by major updates in 2013, 2014, 2017, and 2018. With the most recent changes in 2018, LI-RADS was integrated into the American Association for the Study of Liver Disease (AASLD) practice guidance for HCC $^{[6]}$.

Currently, LI-RADS has four individual algorithms that are used in different clinical contexts. Ultrasound LI-RADS is used for surveillance of at-risk patients. Contrast-enhanced ultrasound LI-RADS is used for the diagnosis of HCC. Computed tomography/magnetic resonance imaging (CT/MRI) LI-RADS algorithm is used for diagnosis and staging of HCC. Treatment response LI-RADS algorithm is used following localregional therapy. This manuscript focuses on LI-RADS CT/MRI diagnostic algorithm.

\section{CT/MRI DIAGNOSTIC ALGORITHM}

LI-RADS CT/MRI Diagnostic Algorithm version 2018 (v2018) includes eight diagnostic categories which are applied to individual observations with increasing probability of HCC and malignancy with higher categories. The various categories do not have an exact correlation to the histologic categories but instead reflect the probability of individual observations being benign, HCC, or non-HCC malignancy. For instance, although all observations in LR-1 are benign and all observations in LR-5 are HCC, the opposite is not true, as many benign lesions may not be categorized LR-1 and not all HCC meet criteria for the LR-5 category ${ }^{[9]}$.

A multiphase contrast-enhanced study is required for appropriate assessment of liver observations. Multiphase studies include late arterial phase, portal venous phase, and delayed phase. Extracellular contrast agents are used for CT exams, while either an extracellular or hepatobiliary agent may be used for MRI ${ }^{[7]}$. Extracellular MRI contrast agents provide lesion characteristics based primarily on blood flow, while hepatobiliary agents utilize information from both blood flow and the hepatocellular function ${ }^{[6]}$. Although recent meta-analyses studies have shown multiphase contrast-enhanced MRI to have a higher sensitivity than CT for the diagnosis of HCC with similar specificity, there is currently insufficient evidence to recommend one modality or contrast agent over the other ${ }^{[6,7,10]}$. Practitioners and institutions are encouraged to make decisions based on their best judgement, as well as to develop their own approach based on a multidisciplinary consensus that would be best suited both for the individual patient and the institution as a whole ${ }^{[6]}$. In treatment-naïve patients, the unenhanced phase on CT is optional, while it is required in patients following loco-regional therapy ${ }^{[7]}$. Late arterial phase (AP) imaging is strongly preferred over the early AP to improve detection of the arterial phase hyperenhancement, an imaging feature that is required for imaging diagnosis of $\mathrm{HCC}^{[7]}$. 
LI-RADS CT/MRI diagnostic algorithm is equally applicable to CT and MRI. It is important to note, however, that the assigned categories may be discordant between the two modalities. Several studies have demonstrated that the LI-RADS categories are discordant between CT and MRI in about 35\%-70\% of cases $^{[9]}$. MRI categorizes more benign lesions as LR-1 compared to CT (25\%-30\% of those lesions were categorized as LR-3 on CT $)^{[11,12]}$. When excluding the LR-1 category, MRI-assigned categories are higher compared to the CT-assigned categories (12\%-31\% of LR-5 observations on MRI were categorized as LR-4, $12 \%$ as LR-3, and $15 \%-29 \%$ were not seen on CT $)^{[9,11,12]}$. The discrepancies in category assignment is likely multifactorial, and they are related to inherent differences between the modalities, inter-reader disagreements, and the fact that some of the imaging features are only assessable by MRI.

To maintain high specificity of the HCC diagnosis, the LI-RADS algorithm must be applied to a welldefined at-risk target population with a high pretest probability of $\mathrm{HCC}^{[7,13]}$. It is essential that the patients meet the inclusion criteria for LI-RADS patient population, and have none of the exclusion criteria. The LI-RADS patient population includes patients with cirrhosis, chronic hepatitis B with or without cirrhosis, and a personal history of $\mathrm{HCC}^{[7,13]}$. The exclusion criteria include patients $<18$ years of age, those with vascular causes of cirrhosis, or those with congenital hepatic fibrosis ${ }^{[8]}$.

The LI-RADS CT/MRI algorithm is applied in a stepwise fashion beginning with LR-NC ${ }^{[7]}$. This is followed by LR-TIV, LR-1, LR-2, and LR-M. Once the above categories are excluded, the CT/MRI diagnostic table is then used to assign an observation LR-3, LR-4, or LR-5 category. Observations with a pathological diagnosis are reported as such and not assigned a LI-RADS category to avoid confusion and uncertainty (i.e., pathology proven intrahepatic cholangiocarcinoma or pathology proven hemangioma). The exception to this is in pathological proven benign or premalignant hepatocellular lesions such as regenerative or dysplastic nodules, which are assigned LI-RADS categories ${ }^{[9]}$. The rationale is that such lesions may evolve over time and progress to frank malignancy.

The CT/MRI diagnostic table uses a combination of the major features [Figure 1] to assign LR-3, LR-4, and LR-5 categories, with the option of applying ancillary features (AF) to adjust the final category. The LI-RADS category can be upgraded or downgraded by one, if there are more than one AF favoring malignancy or benignity, respectively ${ }^{[14]}$. It is important to note that an LR-4 observation cannot be upgraded to LR-5 based on $\mathrm{AF}^{[14]}$. The category is also not adjusted where there are ancillary features favoring both benignity and malignancy ${ }^{[14]}$.

\section{CT/MRI diagnostic categories}

\section{LR-NC: not categorizable}

Observations are included in this category when they cannot be meaningfully categorized because of image omission and/or degradation, preventing assessment of one or more of its major features resulting in the inability to differentiate categories in which cancer is unlikely (LR-1 or LR-2) from categories in which cancer is likely (LR-4, LR-5, and LR-M ${ }^{[7,15]}$. LR-NC should be applied only to observations that are specifically affected by the limitation and not applied to the entire liver ${ }^{[15]}$. Management in this category includes a repeat diagnostic imaging usually within three months with the option of using an alternative modality or contrast agent to improve diagnostic quality ${ }^{[16]}$.

\section{LR-TIV: definite tumor in vein}

Observations in this category demonstrate definite enhancing soft tissue in vein, regardless of the presence of parenchymal mass [Figure 2] ${ }^{[7,15]}$. Approximately $92 \%$ of observations are malignant ${ }^{[17]}$. Approximately $80 \%$ of LR-TIV is attributed to HCC, and some intrahepatic cholangiocarcinoma (iCCA) and combined HCC-cholangiocarcinoma (cHCC-CCA) may also cause tumor in vein ${ }^{[17]}$. When a LR-5 parenchymal observation is associated with TIV, it is reported as "LR-TIV, definitely due to HCC", and, in the absence of 
CT/MRI Diagnostic Table

\begin{tabular}{|c|c|c|c|c|c|c|}
\hline \multirow{2}{*}{\multicolumn{2}{|c|}{$\begin{array}{l}\text { Arterial phase hyperenhancement (APHE) } \\
\text { Observation size }(\mathrm{mm})\end{array}$}} & \multicolumn{2}{|c|}{ No APHE } & \multicolumn{3}{|c|}{ Nonrim APHE } \\
\hline & & $<20$ & $\geq 20$ & $<10$ & $10-19$ & $\geq 20$ \\
\hline \multirow{3}{*}{$\begin{array}{l}\text { Count additional major features: } \\
\text { •Enhancing "capsule" } \\
\text {-Nonperipheral "washout" } \\
\text {-Threshold growth }\end{array}$} & None & LR-3 & LR-3 & LR-3 & LR-3 & LR-4 \\
\hline & One & LR-3 & LR-4 & LR-4 & $-R-4$ LR-5 & LR-5 \\
\hline & $\geq$ Two & LR-4 & LR-4 & LR-4 & LR-5 & LR-5 \\
\hline
\end{tabular}

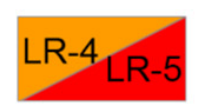

Observations in this cell are categorized based on one additional major feature:

-LR-4 - if enhancing "capsule"

- LR-5 - if nonperipheral "washout" OR threshold growth

\section{If unsure about the presence of any major feature: characterize that feature as absent}

Figure 1. Liver Imaging Reporting and Data System computed tomography/magnetic resonance imaging diagnostic table, used to assign LR-3, LR-4, and LR-5 categories. Reprinted with permission from Ref. ${ }^{[8]}$
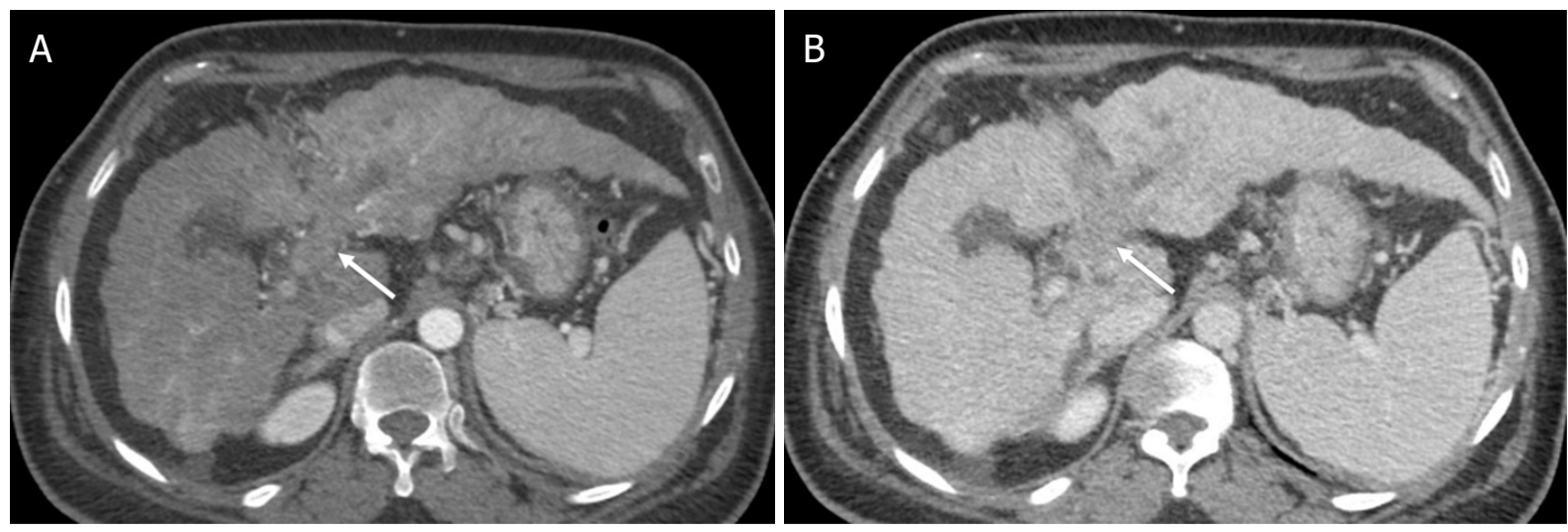

Figure 2. LR-TIV (Definite tumor in vein). Axial computed tomography in a 63-year-old woman with hepatitis C cirrhosis. Arterial phase (A); and portal venous phase (B) demonstrate definite enhancing soft tissue extending to the left portal vein (arrow). The observation is categorized LR-TIV. Note that the presence of parenchymal mass is not required for this category

a discernable parenchymal mass, it is reported as "LR-TIV, probably due to HCC" ${ }^{[9]}$. Occasionally, TIV may be associated with a targetoid parenchymal mass, in which case the category is reported as "LR-TIV, may be due to non-HCC malignancy" ${ }^{\text {"[9] }}$. Management in this category requires multidisciplinary discussion and may require a biopsy if the tumor in vein is not definitely due to $\mathrm{HCC}^{[16,18]}$.

\section{LR-1: definitely benign}

Observations in this category have a $100 \%$ certainty of being benign [Figure 3$]^{[7,15]}$. Observations in this category are usually benign non-hepatocellular lesions or vascular pseudolesions and include cysts, hemangiomas, focal fatty deposition, or sparing and perfusion related changes ${ }^{[9]}$. Benign lesions when evaluated by MRI are more often categorized as LR-1, compared to $\mathrm{CT}^{[1,12]}$. Management of observations in this category involves routine surveillance in six months ${ }^{[16,18]}$.

\section{LR-2: probably benign}

Observations in this category have a high probability but lack $100 \%$ certainty of being benign [Figure 4$]^{[7,15]}$. A recent systematic review showed about $13 \%$ of observations in this category to be HCC and $14 \%$ of the 

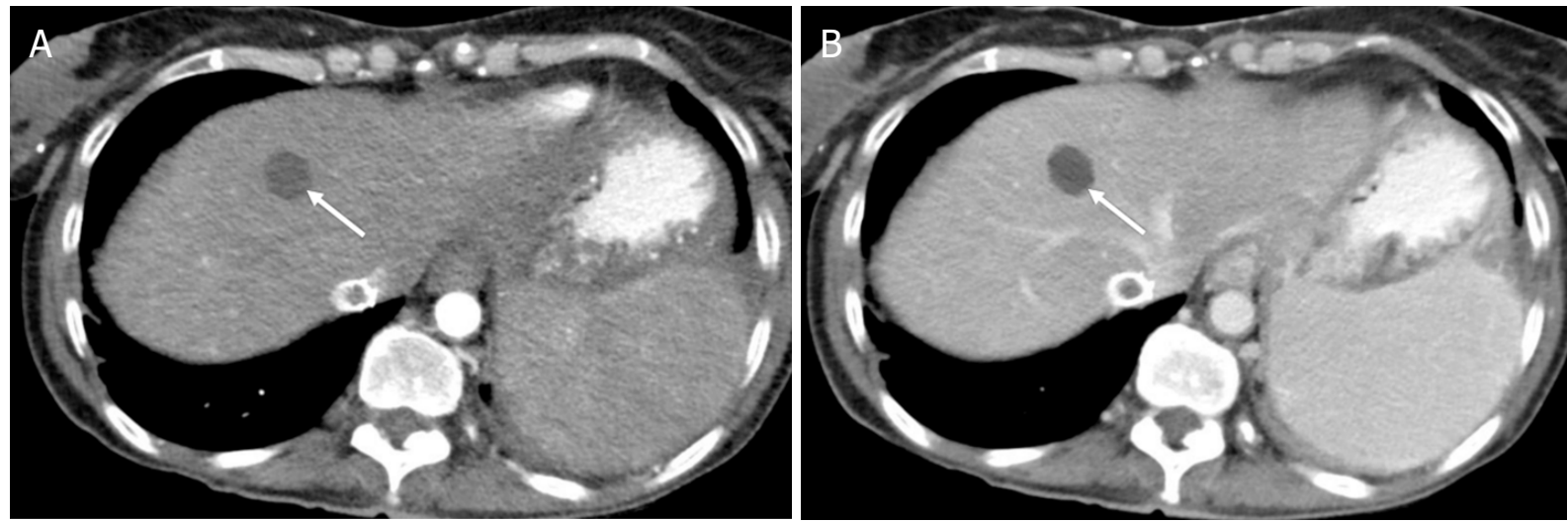

Figure 3. LR-1 (Definitely benign). Axial computed tomography in a 53-year-old woman with hepatitis C cirrhosis. Arterial phase (A); and portal venous phase (B) demonstrate a 17- $\mathrm{mm}$ well-defined round observation (arrow) with attenuation values of simple fluid, consistent with a definite simple cyst
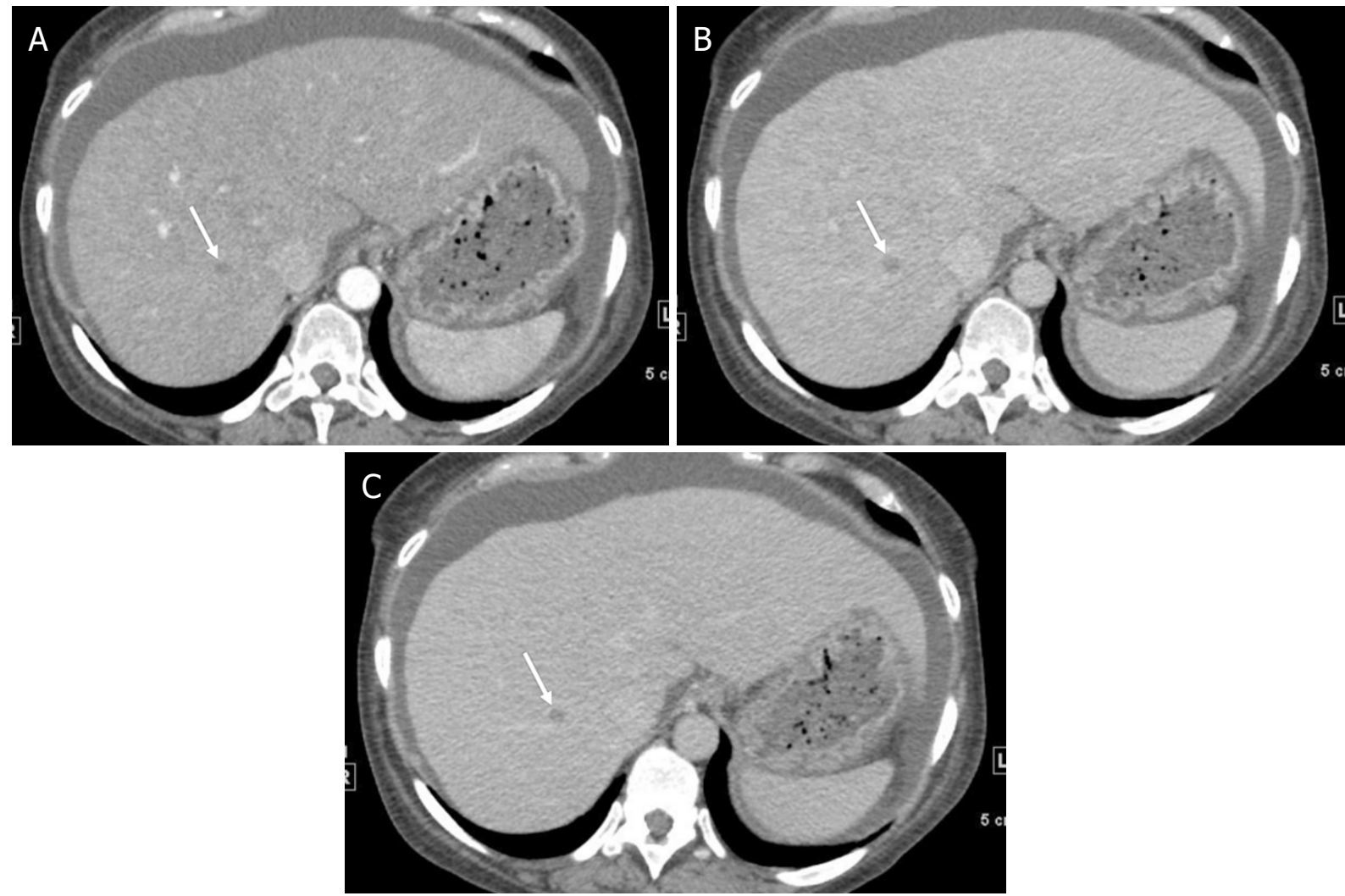

Figure 4. LR-2 (Probably benign). Axial computed tomography in a 46-year-old woman with alcoholic cirrhosis. Arterial phase (A); portal venous phase (B); and delayed phases (C) demonstrate an $8-\mathrm{mm}$ well-defined hypodense observation. The observation is too small to definitively characterize, but probably represents a small cyst

observations to be malignant ${ }^{[17]}$. The majority of the observations in this category are the same as in LR-1 but display atypical imaging features, which result in less than $100 \%$ certainty of making the diagnosis. Additionally, distinctive nodules less than $20 \mathrm{~mm}$ without major imaging features of HCC, features of LR-M, and ancillary features favoring malignancy are categorized LR- ${ }^{[9]}$. LR-2 distinctive nodules include siderotic nodules, T1-hyperintense nodules, T2-hypointense nodules, and nodules hyperintense on hepatobiliary phase. Some LR-3 observation can be down-categorized to LR-2, if there are ancillary 

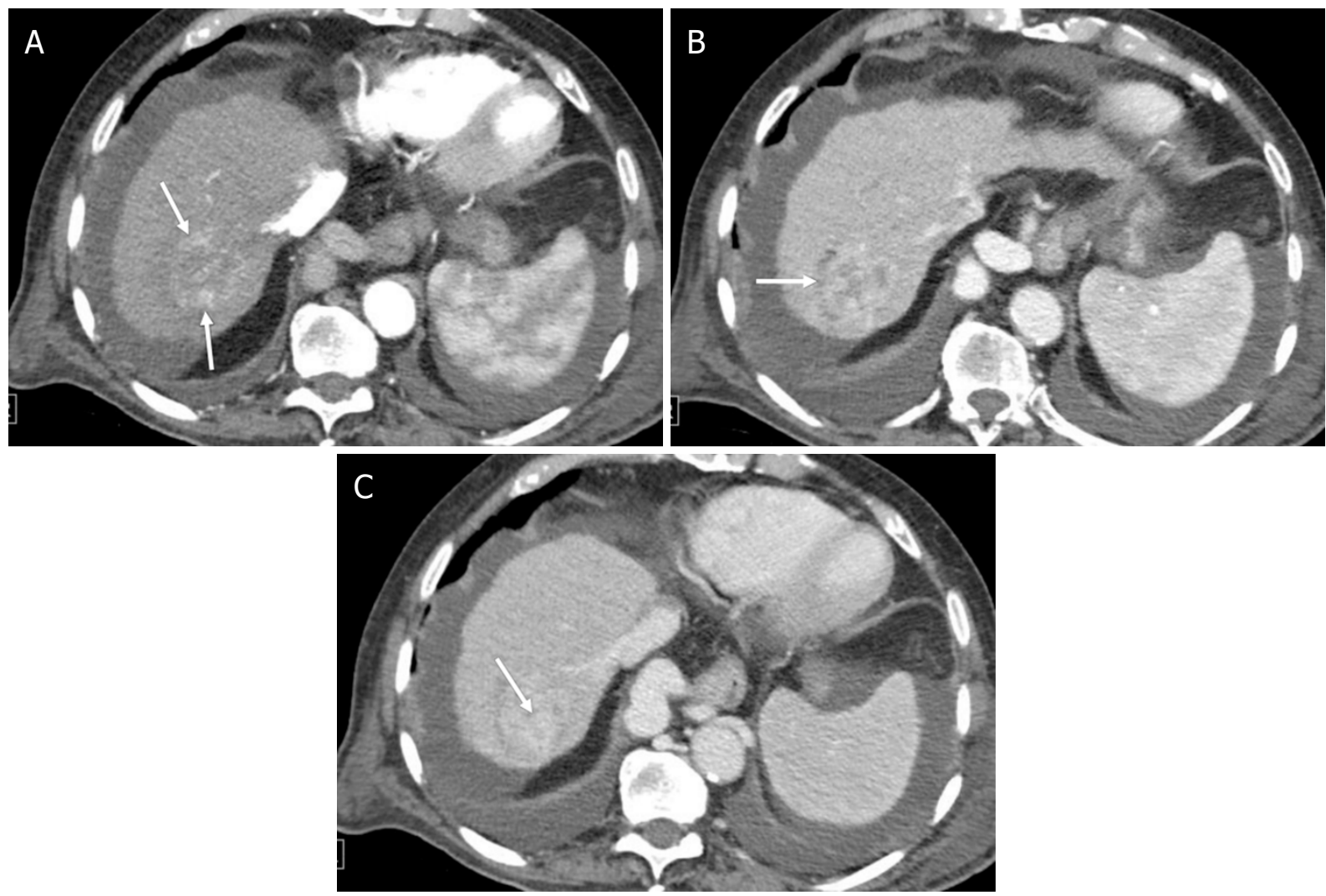

Figure 5. LR-M (Probably or definitely malignant, not hepatocellular carcinoma specific). Axial Computed Tomography in an 80-year-old man with hepatitis C cirrhosis. Arterial phase (A); portal venous phase (B); and delayed phases (C) demonstrate a 40-mm observation with rim arterial phase hyperenhancement [arrows (A)], peripheral washout appearance [arrow (B)], and delayed central enhancement [arrow (C)] observation. Subsequent biopsy confirmed an intrahepatic cholangiocarcinoma

features favoring benignity and no ancillary features of malignancy ${ }^{[9]}$. Management in LR-2 category usually includes routine surveillance in six months; however, occasionally repeat diagnostic imaging with a different modality in six months and/or multimodality discussion may be warranted ${ }^{[16,18]}$.

\section{LR-M: probably or definitely malignant, not HCC specific}

Observations in this category have a high probability or $100 \%$ certainty of being malignant but the features are not specific for HCC [Figure 5] ${ }^{[7,15]}$. Approximately $93 \%$ of observations in this group are malignant, with non-HCC malignancies such as iCCA, cHCC-CCA, and metastases comprising the majority of the LR-M category and HCC accounting for $36 \%$ of the observations in this group ${ }^{[17]}$. On rare occasions, benign lesions such as sclerosed hemangiomas may have features that meet the criteria for LR-M. Both targetoid and non-targetoid lesions are included in this category. Targetoid lesions include observations with a targetoid morphology, such as targetoid dynamic enhancement pattern, targetoid diffusion restriction, and targetoid transitional or hepatobiliary phase hypointensity ${ }^{[19]}$. Non-targetoid lesions in the LR-M group include observations that do not meet the criteria for LR-5 or LR-TIV and with at least one of the following features: infiltrative appearance, marked restricted diffusion, necrosis or severe ischemia, or other features suggesting non-HCC malignancy ${ }^{[19]}$. Management requires multidisciplinary discussion and often a biopsy is needed for diagnosis, staging, and management ${ }^{[16,18]}$.

\section{LR-3: intermediate probability of malignancy}

Observations in this category include malignant and nonmalignant lesions, resulting in a moderate probability of being malignant [Figure 6$]^{[7,15]}$. Forty percent of observations in this category are malignant 

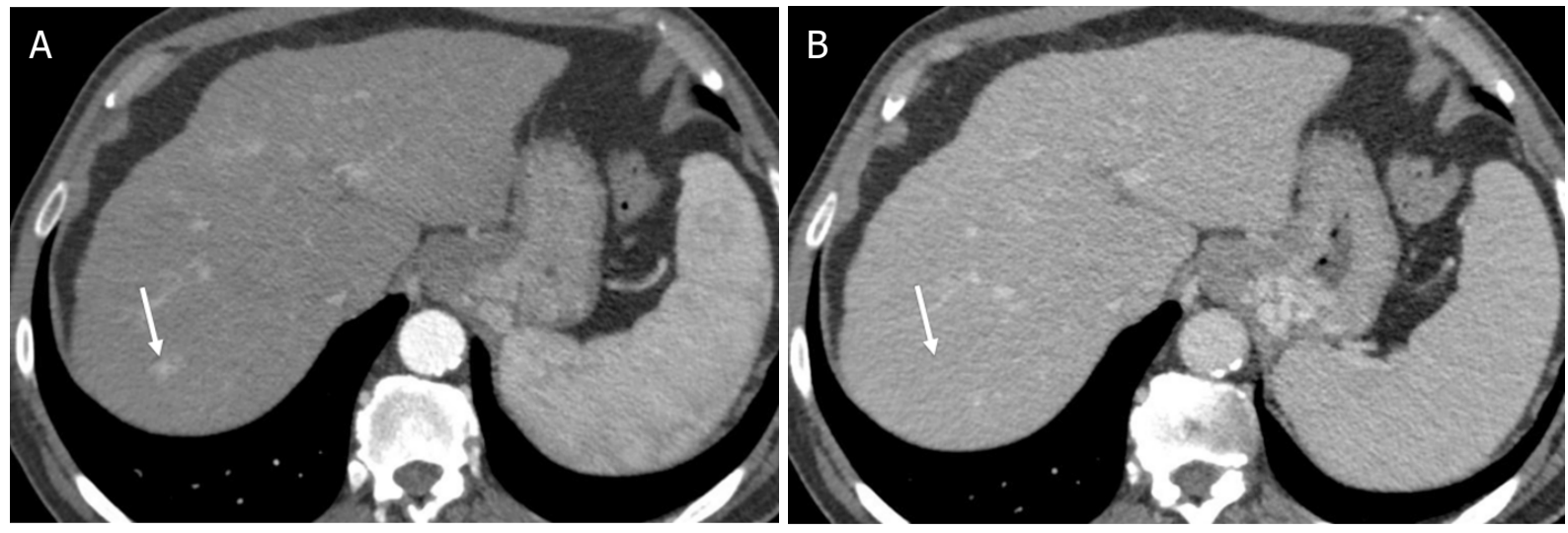

Figure 6. LR-3 (Intermediate probability hepatocellular carcinoma). Axial computed tomography in a 68-year-old man with hepatitis $C$ cirrhosis. Arterial phase (A); and portal venous phase (B) demonstrate a 12-mm observation with nonrim arterial phase hyperenhancement [arrow (A)] and no washout appearance or enhancing capsule appearance [arrow (B)]
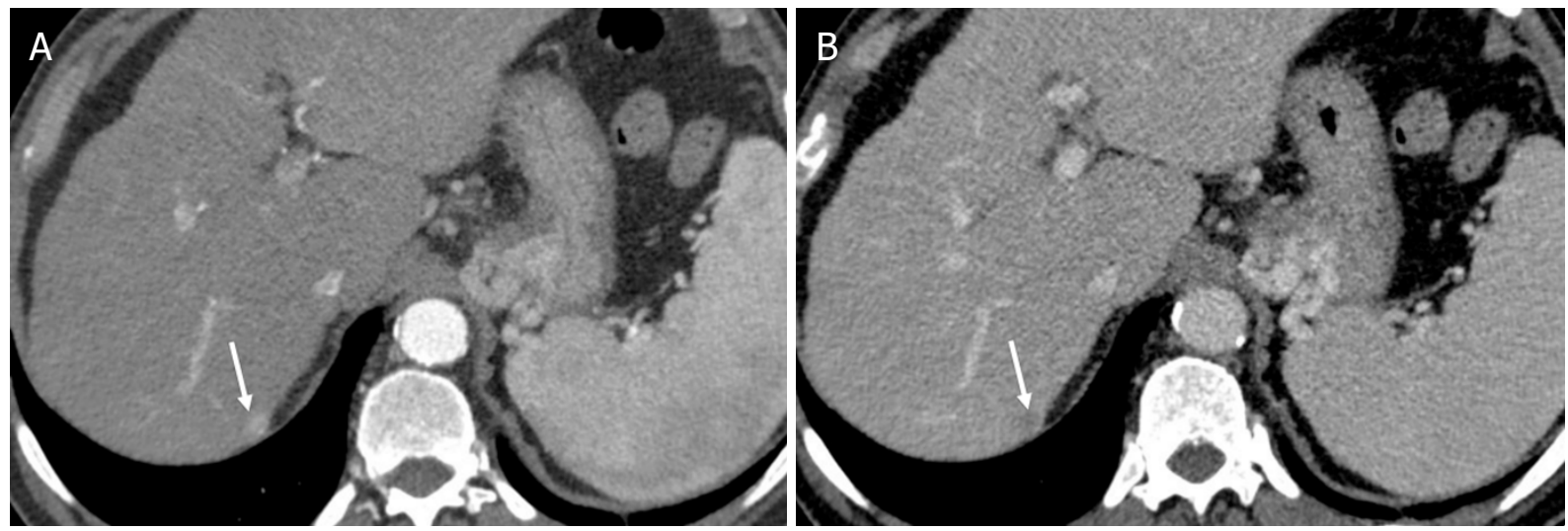

Figure 7. LR-4 (Probable hepatocellular carcinoma). Axial computed tomography in a 68-year-old man with hepatitis C cirrhosis. Arterial phase (A); and portal venous phase (B) demonstrate a 9-mm observation with nonrim arterial phase hyperenhancement [arrow $(A)]$, nonperipheral washout appearance [arrow (B)], and no enhancing capsule appearance

with $38 \%$ being $\mathrm{HCC}^{[17]}$. Observations $<20 \mathrm{~mm}$ demonstrating nonrim arterial phase hyperenhancement (APHE) alone are categorized in this category ${ }^{[7,15]}$. Observations without APHE can also be categorized in this group if they are $<20 \mathrm{~mm}$ with $\leq 1$ additional major feature, or if the observations are $\geq 20 \mathrm{~mm}$ with no additional major features ${ }^{[7,15]}$. LR- 4 observations can be down-categorized to LR-3 if there are $\geq 1$ AF of benignity ${ }^{[7,15]}$. Management options include repeat diagnostic imaging in 3-6 months with or without the use of an alternative modality or contrast agent and occasionally a multidisciplinary discussion may be warranted $^{[16,18]}$.

\section{LR-4: probably HCC}

Observations in this category have a high probability but not $100 \%$ certainty of being HCC [Figure 7$]^{[7,15]}$. The probability of observations being HCC in this category approaches $75 \%-80 \%{ }^{[17,20]}$. Observations in this category include observations $<10 \mathrm{~mm}$ with nonrim APHE and $\geq 1$ additional major feature, observations that are 10-19 mm with nonrim APHE and enhancing "capsule" as the only major feature, and observations $\geq 20 \mathrm{~mm}$ with nonrim APHE and no additional major feature. Observations without nonrim APHE can be categorized LR- 4 with the size $<20 \mathrm{~mm}$ and $\geq 2$ additional major features or with size $\geq 20 \mathrm{~mm}$ and $\geq 1$ additional major feature ${ }^{[7,15]}$. LR-3 observations with $\geq 1$ AF favoring malignancy can be upgraded to LR-4, while LR- 5 observations with $\geq 1$ AF favoring benignity can be downgraded to LR- $4^{[9]}$. Management often 

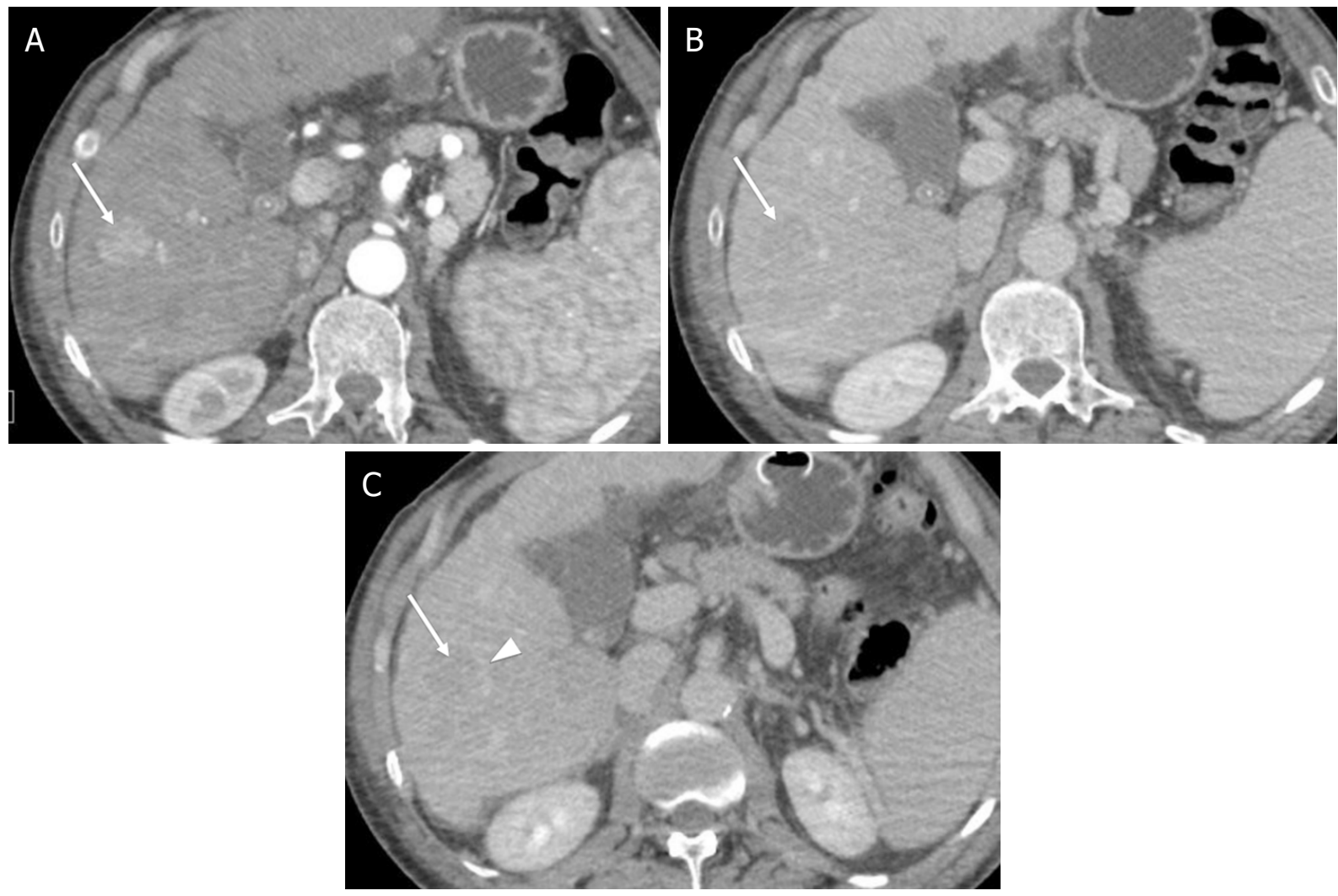

Figure 8. LR-5 (Definite hepatocellular carcinoma). Axial computed tomography in a 60-year-old man with hepatitis $C$ cirrhosis. Arterial phase (A); portal venous phase (B); and delayed phases (C) demonstrate a 21-mm observation with nonrim arterial phase hyperenhancement [arrow (a)], nonperipheral washout appearance [arrow $(B, C)]$, and enhancing capsule appearance [arrowhead (c)]

involves multidisciplinary discussion and may include repeat or alternate imaging within three months, biopsy, or, in some patients, definitive treatment without confirmatory biopsy ${ }^{[16,18]}$.

\section{LR-5: definitely HCC}

Observations in this category have $100 \%$ certainty of being HCC [Figure 8$]^{[7,15]}$. Ninety-four percent of observations in this category are HCC and $97 \%$ are malignant ${ }^{[17]}$. Since HCC can be diagnosed based on imaging features alone and treated without the need for pathologic confirmation, observations in this category have specificity close to $100 \%$ with resulting modest sensitivity in the range of $50 \%-80 \%{ }^{[21-23]}$. Size $\geq$ $10 \mathrm{~mm}$ and nonrim APHE are absolute requirements to LR-5 categorization ${ }^{[7,15]}$. Observations measuring > $20 \mathrm{~mm}$ with $\geq 1$ additional major feature and observations measuring 10-19 $\mathrm{mm}$ with $\geq 2$ additional major features are included in this category ${ }^{[7,15]}$. Observations measuring $10-19 \mathrm{~mm}$ with either nonperipheral "washout" or threshold growth as the only additional major feature are also categorized LR-5. ${ }^{[7,15]}$. Ancillary features cannot be used to upgrade LR- 4 observations to LR- 5 in order to maintain the required high specificity in this category ${ }^{[7,15]}$. Management includes multidisciplinary discussion for staging and treatment without the need for a biopsy ${ }^{[16,18]}$. In some patients, a tissue sample may be required for histologic grading, molecular characterization, or enrollment in clinical trials ${ }^{[16,18]}$.

\section{INTEGRATION INTO AASLD}

LI-RADS has undergone several major updates since its initial release in 2011. The most recent update (LI-RADS v2018) has revised its criteria for LR-5 (definite HCC), and as a result LI-RADS has been integrated into the AASLD HCC practice guidelines and simplified ${ }^{[6]}$. In v2018, 10-19-mm observations with nonrim APHE and nonperipheral "washout" are categorized LR-5, regardless of visualization on the 
antecedent surveillance ultrasound ${ }^{[7,8]}$. Additionally, v2018 updated the definition for threshold growth to be congruent with that of the Organ Procurement and Transplantation Network: threshold growth is defined as $\geq 50 \%$ increase in size of a mass in $\leq 6$ months $^{[8]}$. Any other size increase, including new observations and observations with $\geq 100 \%$ increase in size on studies $>6$ months apart, are characterized subthreshold growth ${ }^{[8]}$.

\section{LI-RADS: ADVANTAGES FOR CLINICIANS}

As the diagnosis of HCC is often made based on imaging findings alone in patients at risk without the need for a biopsy confirmation, it is imperative to maintain a high level of accuracy among radiologists in both the interpretation and the reporting of liver observations ${ }^{[24-26]}$. LI-RADS uses a strict diagnostic criterion and an algorithmic approach to determine the likelihood of focal liver observations being HCC, while maintaining substantial inter-reader reliability and a high specificity for $\operatorname{HCC}^{[17,27,28]}$. In fact, LI-RADS interreader agreement on MRI compares favorably to other commonly used RAD systems, such as breast and $\operatorname{prostate}^{[29]}$.

The use of non-standardized lexicon in liver imaging reports may result in confusion, especially when communicating results to the referring clinicians ${ }^{[30]}$. The same phrase may be used differently by different radiologists and may be interpreted differently by the referring clinicians ${ }^{[31]}$. Corwin et al ${ }^{[31]}$ reported that the use of radiology phrases such as "consistent with HCC" and "suspicious for HCC" were associated with a high variability in the LI-RADS categories. Conversely, use of LI-RADS terminology and reporting results in a more comprehensive, clearer, and ultimately more actionable report ${ }^{[32]}$.

\section{DISCUSSION}

While LI-RADS offers numerous advantages for radiologists, clinicians, and patients, the system is not perfect. Unlike many other imaging-based systems for HCC diagnosis which have binary approach for diagnosis (i.e., HCC vs. all other lesions), LI-RADS utilizes ordinal categories which reflect increasing probability of HCC. Furthermore, LI-RADS is unique in that it recognizes the increased risk of non-HCC malignancies in patients with cirrhosis and provides diagnostic criteria for LR-M category. This level of detail requires greater complexity of the diagnostic criteria, and as a result LI-RADS may be intimidating for novice users or may be perceived as too cumbersome for clinical practice. However, a recent survey of radiologists and clinicians found that nearly $90 \%$ preferred the use of LI-RADS compared to other standardized reporting systems ${ }^{[33]}$.

LI-RADS has undergone several major updates since its original release in 2011. As scientific evidence accumulates, and user feedback is accrued, the criteria are refined and updated ${ }^{[7]}$. The goal of each release is to improve accuracy and ease of use $\mathrm{e}^{[7]}$. The need for the system to be in congruence with the latest scientific knowledge is balanced against the need for a stable system $^{[7]}$. As a result, major updates to the system are planned for every 3-5 years.

\section{CONCLUSION}

This manuscript reviews CT/MRI LI-RADS. CT/MRI LI-RADS is a comprehensive and dynamic system with standardized terminology and an algorithmic diagnostic approach for the diagnosis and subsequent management of HCC, enabling clear communication between radiologist and clinicians. The most recent version has revised its criteria for LR-5 and simplified its definition for threshold growth, and it is now incorporated into the AASLD HCC practice guidelines.

\section{DECLARATIONS}

\section{Authors' contributions}

Made substantial contributions to drafting, editing and finalizing of the draft: Kanmaniraja D, Chernyak V 


\section{Availability of data and materials}

Not applicable.

\section{Financial support and sponsorship}

None.

\section{Conflicts of interest}

Chernyak V is the consultant for Bayer; Kanmaniraja D declared that there are no conflicts of interest.

\section{Ethical approval and consent to participate}

Not applicable.

\section{Consent for publication}

Not applicable.

\section{Copyright}

(c) The Author(s) 2020.

\section{REFERENCES}

1. GLOBOCAN International Agency for Research on Cancer (IARC). Available from: https://gco.iarc.fr/. [Last accessed on 7 July 2020 ]

2. World Health Organization Mortality Database. WHO Statistical Information System. 2008. Available from: https://www.who.int/newsroom/fact-sheets/detail/cancer. [Last accessed on 7 July 2020].

3. Petrick JL, Kelly SP, Altekruse SF, McGlynn KA, Rosenberg PS. Future of hepatocellular carcinoma incidence in the United States forecast through 2030. J Clin Oncol 2016;34:1787-94.

4. Petrick JL, Braunlin M, Laversanne M, Valery PC, Bray F, et al. International trends in liver cancer incidence, overall and by histologic subtype, 1978-2007. Int J Cancer 2016;139:1534-45.

5. Tang A, Bashir MR, Corwin MT, Cruite I, Dietrich CF, et al. Evidence supporting LI-RADS major features for CT- and MR imagingbased diagnosis of hepatocellular carcinoma: a systematic review. Radiology 2018;286:29-48.

6. Marrero JA, Kulik LM, Sirlin CB, Zhu AX, Finn RS, et al. Diagnosis, staging, and management of hepatocellular carcinoma: 2018 Practice Guidance by the American Association for the Study of Liver Diseases. Hepatology 2018;68:723-50.

7. Chernyak V, Fowler KJ, Kamaya A, Kielar AZ, Elsayes KM, et al. Liver imaging reporting and data system (LI-RADS) Version 2018: imaging of hepatocellular carcinoma in at-risk patients. Radiology 2018;289:816-30.

8. The American College of Radiology LI-RADS v2018 Core. Available from: https://www.acr.org/-/media/ACR/Files/RADS/LI-RADS/LIRADS-2018-Core.pdf?la=en. [Last accessed on 29 Jun 2020]

9. Chapter-8-LIRADS-Categories. ACR. Available from: https://www.acr.org/-/media/ACR/Files/Clinical-Resources/LIRADS/Chapter-8LIRADS-Categories.pdf?la=en. [Last accessed on 29 Jun 2020]

10. Roberts LR, Sirlin CB, Zaiem F, Almasri J, Prokop LJ, et al. Imaging for the diagnosis of hepatocellular carcinoma: a systematic review and meta-analysis. Hepatology 2018;67:401-21.

11. Basha MAA, AlAzzazy MZ, Ahmed AF, Yousef HY, Shehata SM, et al. Does a combined CT and MRI protocol enhance the diagnostic efficacy of LI-RADS in the categorization of hepatic observations? A prospective comparative study. Eur Radiol 2018;28:2592-603.

12. Corwin MT, Fananapazir G, Jin M, Lamba R, Bashir MR. Differences in liver imaging and reporting data system categorization between MRI and CT. AJR Am J Roentgenol 2016;206:307-12.

13. Tang A, Hallouch O, Chernyak V, Kamaya A, Sirlin CB. Epidemiology of hepatocellular carcinoma: target population for surveillance and diagnosis. Abdom Radiol (NY) 2018;43:13-25.

14. Chernyak V, Tang A, Flusberg M, Papadatos D, Bijan B, et al. LI-RADS((R)) ancillary features on CT and MRI. Abdom Radiol (NY) 2018;43:82-100.

15. Santillan C, Chernyak V, Sirlin C. LI-RADS categories: concepts, definitions, and criteria. Abdom Radiol (NY) 2018;43:101-10.

16. Chapter-11-Management. ACR. Available from: https://www.acr.org/-/media/ACR/Files/Clinical-Resources/LIRADS/Chapter-11Management.pdf?la=en. [Last accessed on 29 Jun 2020]

17. van der Pol CB, Lim CS, Sirlin CB, McGrath TA, Salameh JP, et al. Accuracy of the liver imaging reporting and data system in computed tomography and magnetic resonance image analysis of hepatocellular carcinoma or overall malignancy-a systematic review. Gastroenterology 2019;156:976-86.

18. Mitchell DG, Bruix J, Sherman M, Sirlin CB. LI-RADS (Liver Imaging Reporting and Data System): summary, discussion, and consensus of the LI-RADS Management Working Group and future directions. Hepatology 2015;61:1056-65.

19. Fowler KJ, Potretzke TA, Hope TA, Costa EA, Wilson SR. LI-RADS M (LR-M): definite or probable malignancy, not specific for hepatocellular carcinoma. Abdom Radiol (NY) 2018;43:149-57. 
20. Choi SH, Byun JH, Kim SY, Lee SJ, Won HJ, et al. Liver Imaging Reporting and Data System v2014 with gadoxetate disodium-enhanced magnetic resonance imaging: validation of LI-RADS Category 4 and 5 Criteria. Invest Radiol 2016;51:483-90.

21. Chernyak V, Flusberg M, Berman J, Fruitman KC, Kobi M, et al. Liver Imaging Reporting and Data System (LI-RADS) v2018: impact on categorization and hepatocellular carcinoma staging. Liver Transpl 2019;25:1488-502.

22. Lee SM, Lee JM, Ahn SJ, Kang HJ, Yang HK, et al. LI-RADS Version 2017 versus Version 2018: diagnosis of hepatocellular carcinoma on gadoxetate disodium-enhanced MRI. Radiology 2019;292:655-63.

23. Ren AH, Zhao PF, Yang DW, Du JB, Wang ZC, et al. Diagnostic performance of MR for hepatocellular carcinoma based on LI-RADS v2018, compared with v2017. J Magn Reson Imaging 2019;50:746-55.

24. Heimbach JK, Kulik LM, Finn RS, Sirlin CB, Abecassis MM, et al. AASLD guidelines for the treatment of hepatocellular carcinoma. Hepatology 2018;67:358-80.

25. Chernyak V, Santillan CS, Papadatos D, Sirlin CB. LI-RADS((R)) algorithm: CT and MRI. Abdom Radiol (NY) 2018;43:111-26.

26. Tang A, Valasek MA, Sirlin CB. Update on the liver imaging reporting and data system: what the pathologist needs to know. Adv Anat Pathol 2015;22:314-22.

27. Fowler KJ, Tang A, Santillan C, Bhargavan-Chatfield M, Heiken J, et al. Interreader Reliability of LI-RADS Version 2014 algorithm and imaging features for diagnosis of hepatocellular carcinoma: a large international multireader study. Radiology 2018;286:173-85.

28. Kang JH, Choi SH, Lee JS, Park SH, Kim KW, et al. Interreader agreement of liver imaging reporting and data system on MRI: a systematic review and meta-analysis. J Magn Reson Imaging 2020; doi: 10.1002/jmri.27065.

29. Chernyak V, Sirlin CB. Editorial for "Interreader Agreement of Liver Imaging Reporting and Data System on MRI: a systematic review and meta analysis". J Magn Reson Imaging 2020; doi: 10.1002/jmri.27133.

30. Goldberg-Stein S, Chernyak V. Adding value in radiology reporting. J Am Coll Radiol 2019;16:1292-8.

31. Corwin MT, Lee AY, Fananapazir G, Loehfelm TW, Sarkar S, et al. Nonstandardized terminology to describe focal liver lesions in patients at risk for hepatocellular carcinoma: implications regarding clinical communication. AJR Am J Roentgenol 2018;210:85-90.

32. Flusberg M, Ganeles J, Ekinci T, Goldberg-Stein S, Paroder V, et al. Impact of a structured report template on the quality of CT and MRI reports for hepatocellular carcinoma diagnosis. J Am Coll Radiol 2017;14:1206-11.

33. Alenazi AO, Elsayes KM, Marks RM, Yacoub JH, Hecht EM, et al. Clinicians and surgeon survey regarding current and future versions of CT/MRI LI-RADS. Abdom Radiol (NY) 2020;45:2603-11. 